\title{
Primary infertility secondary to a hidden posterior urethral valve representing a diagnostic challenge in the fifth decade
}

Emad Rajih, MD, MPH, Tareef Daqqaq, MD, Abdulrahman Mojallid, MD, Said Kattan, MD, FRCSC.

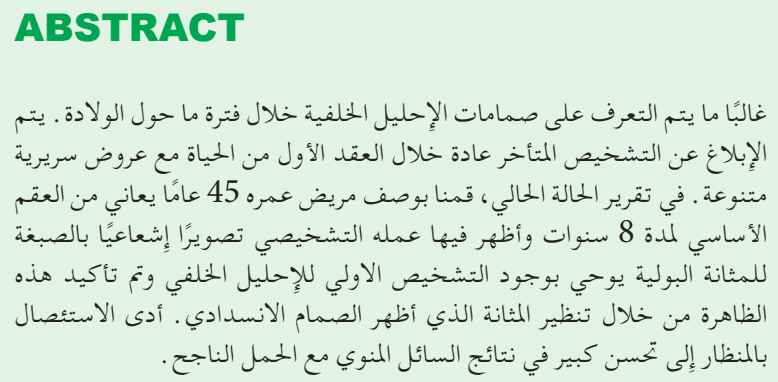

Posterior urethral valves (PUVs) are frequently recognized during the perinatal period. Delayed diagnosis is reported usually within the first decade of life with diverse clinical presentations. In the current case report, we describe a 45 years old man patient who presented with aspermia and primary infertility for 8 years in whom his diagnostic workup revealed radiological imaging suggestive of PUVs. This phenomenon was confirmed by cystourethroscopy that showed obstructive valve. Endoscopic ablation resulted in significant improvement of his seminal parameter with successful conception.

Keywords: posterior urethral valves, fertility, urethral obstruction

Saudi Med J 2020; Vol. 41 (9): 1011-1014

doi: 10.15537/smj.2020.9.25229

From the Urology Department (Rajih); Radiology Department (Daqqaq), College of Medicine, Taibah University; from the Urology Department (Rajih), King Fahad General Hospitals, Madinah; and from the Urology Department (Kattan), King Faisal Specialist Hospital \& Research Center, Riyadh, Kingdom of Saudi Arabia.

Received 18th May 2020. Accepted 7th July 2020.

Address correspondence and reprint request to: Dr. Emad Rajih, Associate Professor, Male Genital Reconstruction/Sexual Medicine \& Minimal invasive Urology, College of Medicine, Taibah University, Madinah, Kingdom of Saudi Arabia.E-mail: remad@taibahu.edu.sa

ORCID ID: https://orcid.org/0000-0002-4641-8248
Dosterior urethral valves (PUVs) are a common 1 cause of infravesical obstruction in male neonates, with an incidence of about one case per 8,000 to 25,000 live births. ${ }^{1}$ With the advancement in antenatal ultrasonography and screening, the diagnosis and early detection of PUVs have been improved in the developed countries. Less frequently, approximately $10 \%$ of the cases are recognized after birth within the first decade, with usual clinical presentations that include weak stream, recurrent infections, gross hematuria, structural anatomical changes of the urinary tract and renal insufficiency. Diagnostic challenges of PUVs have been stated when not recognized during the perinatal period. ${ }^{2,3}$ Delayed diagnosis impacts patients' quality of life and daily activities. The association of sexual dysfunctions and infertility with PUVs has rarely been reported. ${ }^{4}$ They are mainly attributed to the associated medical conditions such as cryptorchidism, renal insufficiency and hypertension. Seminal fluid abnormalities and associated genital organ malformations can be secondarily affected by distal urethral obstruction. ${ }^{4}$ In this report, we describe the diagnostic challenges, findings and management outcome in a patient who had a delayed presentation with infertility secondary to PUV. This case report highlights the direct effect of the obstructive valve on the fertility status and it emphasizes about the diagnostic steps in case of male infertility.

Case Report. A 45-year-old African man presented to our clinic with primary infertility for 8 years. He was complaining of weak stream, frequency and nocturia, along with a small ejaculatory semen volume. He had no history suggestive of urogenital infections. He underwent left varicocelectomy and had multiple visits

Disclosure. Authors have no conflict of interests, and the work was not supported or funded by any drug company. 
to different medical centres along with his 27-year-old wife seeking conception. He was well androgenised with no gynecomastia.

Clinical findings. His vital signs were normal, and his abdominal examination revealed an abdominal scare of high venous ligation. He had intact vas bilaterally without epididymal induration, and both testes measured approximately $18 \mathrm{ml}$. The digital rectal examination showed a firm, small, non-tender prostate.

Diagnostic assessment. Serum chemistries were within the normal range, with a urea level of $27 \mathrm{mg} / \mathrm{dL}$ and a creatinine level of $0.9 \mathrm{mg} / \mathrm{dL}$. Semen analysis showed a low ejaculate volume, $1 \mathrm{~mL}$, and azospermia. Hormonal workup was normal. Urine analysis post ejaculation showed no sperms and unremarkable microscopic analysis for blood and pus cell. Uroflowmetry showed maximum flow rate of 12 $\mathrm{ml} / \mathrm{second}$, voided volume of $190 \mathrm{ml}$, and post void residual of $110 \mathrm{ml}$. Transabdominal ultrasonography of the genitourinary tract showed normal renal echogenicity without hydronephrosis and thick urinary bladder wall. Office transrectal ultrasonography showed dilated seminal vesicles and prostatic calcification around the ejaculatory ducts. Magnetic resonance imaging (MRI) showed a thick urinary bladder wall and dilated prostatic urethra and seminal vesicles (Figure 1). Diagnostic office cystoscopy was carried out to assess the voiding dysfunction that was present, along with the MRI findings. Cystoscopy revealed intact verumontanum, dilated prostatic urethra, high and hypertrophied bladder neck, and sever bladder trabeculations. Subsequently, we assessed the functional status by voiding cystourethrogram (VCUG), which showed multiple small bladder diverticula, irregular bladder wall, severely dilated prostatic urethra with stenosis at the membranous urethra and contrast filling of the genital glands through ejaculatory ducts (Figure 2). His bladder capacity was $320 \mathrm{~mL}$.

Therapeutic intervention. At this phase, cystoscopy was repeated at the operative room under spinal anesthesia to look specifically for any obstructive valve by filling the bladder and placing the scope distal to the membranous urethra, which confirmed the presence of type 1 PUV. The valve leaflets arise from the verumontanum. It extends anteriorly to fuse in the midline proximal to the sphincter. Valve ablation was carried out by using the hook of the monopolar resectoscope to ablate the leaflets at 5 and 7 o'clock utilizing the cutting current. No bleeding was encountered during ablation. Foley's catheter was inserted post ablation and removed at day 3 postoperatively. The endoscopic procedure covered by intravenous Gentamycin $80 \mathrm{mg}$ intravenously.

Follow-up and outcomes. Postoperatively, uroflowmetry revealed a maximal flow rate of $20 \mathrm{~mL} / \mathrm{sec}$, with a voided volume of $170 \mathrm{~mL}$ and a minimal residual
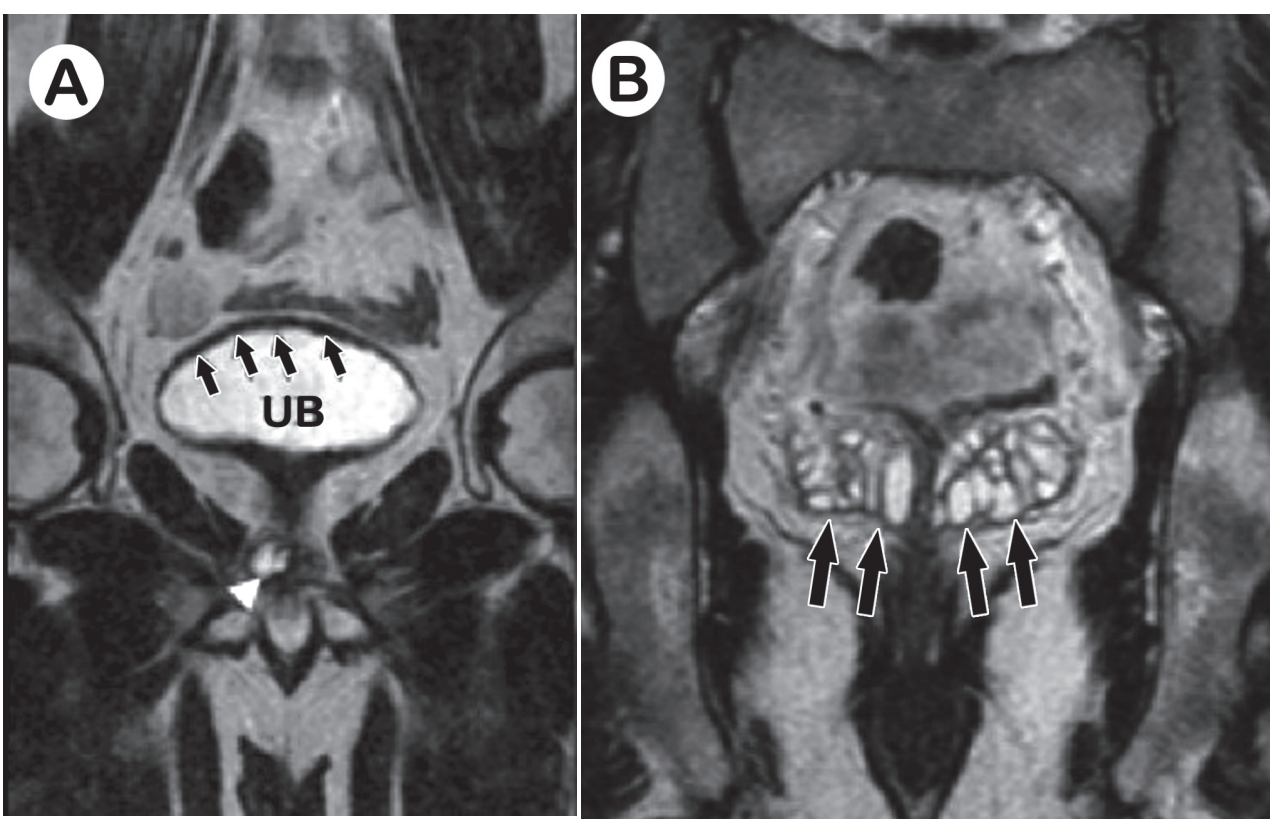

Figure 1 - Coronal T2-weighted MRI image showing A) thick urinary bladder (UB) wall (arrows) and dilated ejaculatory duct (arrowhead) and B) dilated seminal vesicles (arrows). 


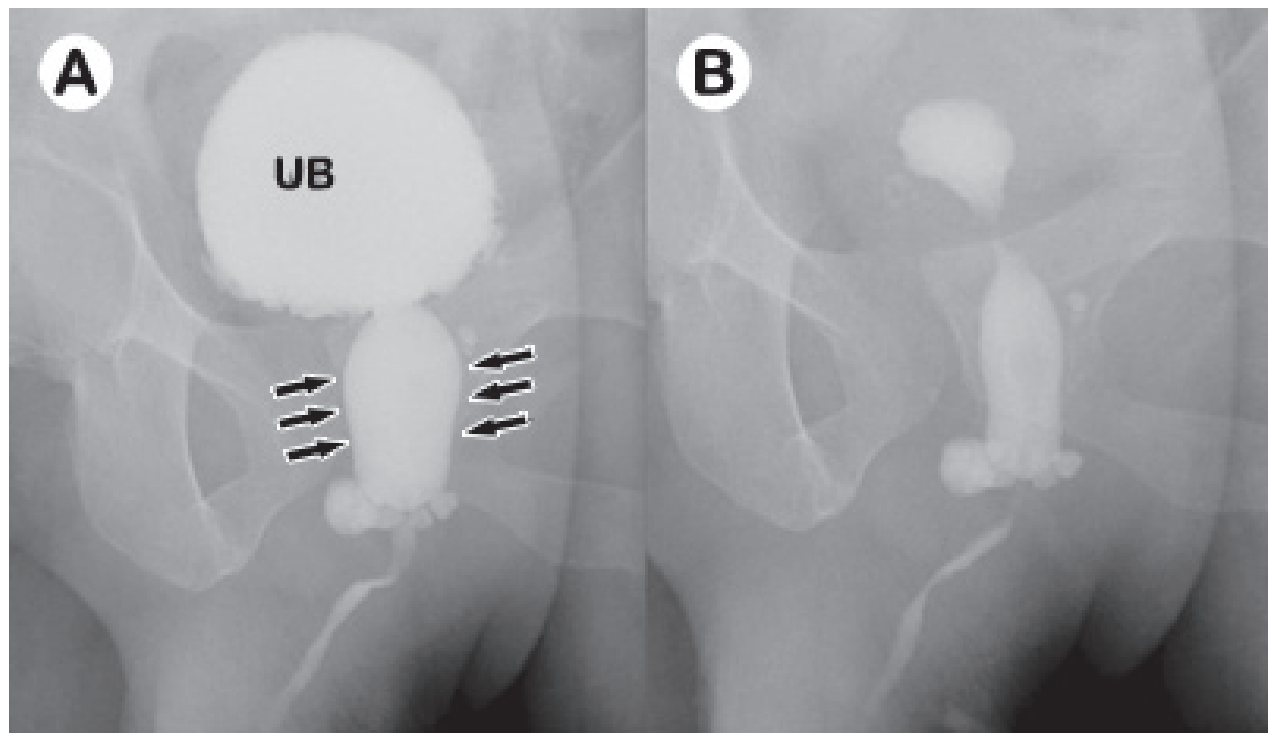

Figure 2 - Voiding cystourethrogram showing dilated prostatic (posterior) urethra (arrows) with abrupt narrowing, along with bladder neck hypertrophy and urinary bladder (UB) wall trabeculations A) Voiding phase B) Postvoid residual.

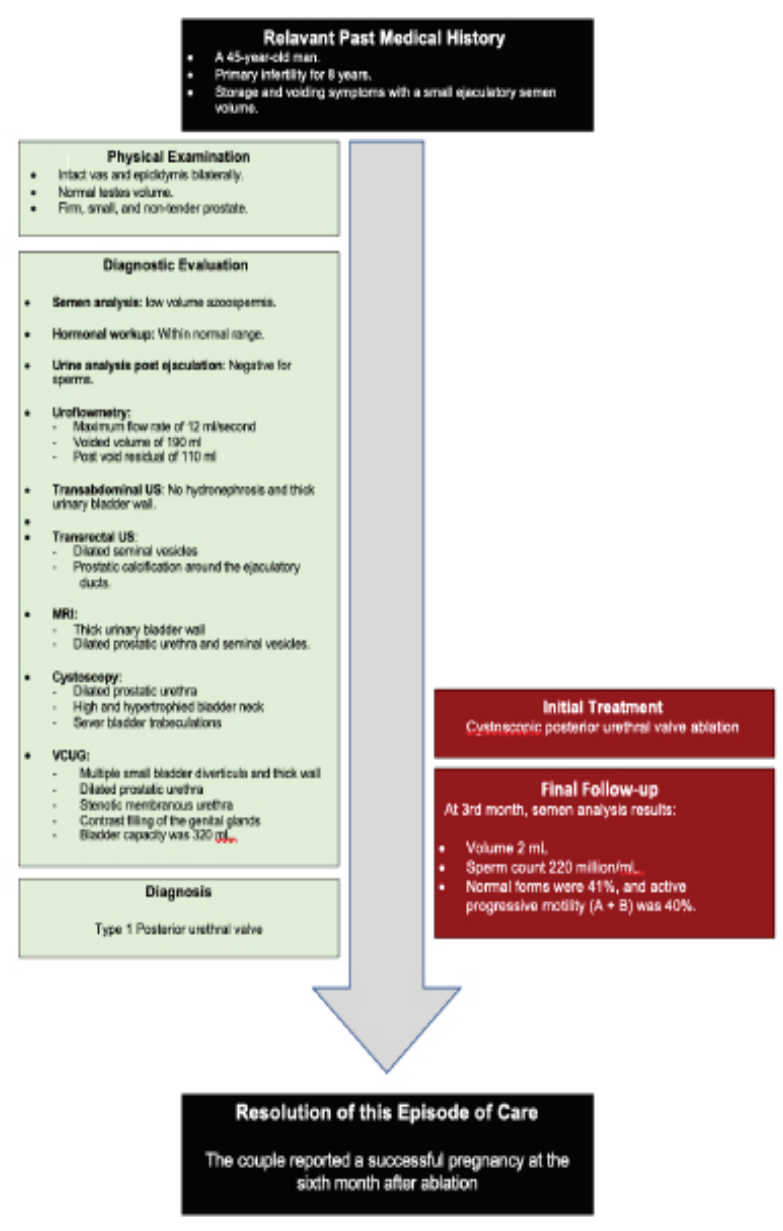

Figure 3 - The sequences of the evaluation process and its integration urinary volume. At 3 months after valve ablation, semen analysis showed a semen volume about $2 \mathrm{~mL}$ and a sperm count of $220 \mathrm{million} / \mathrm{mL}$. Normal forms were $41 \%$, and active motility $(\mathrm{A}+\mathrm{B})$ was $40 \%$. The couple reported a successful pregnancy at the sixth month after ablation. Figure 3 shows the sequences of the evaluation process and its integration.

Discussion. The current report presents a case of PUV that was diagnosed lately in the fifth decade of life. This condition was diagnosed while evaluating the primary infertility with obstructive azoospermia. The PUV was suspected during assessments with the VCUG findings, which was carried out to evaluate the voiding dysfunction that was associated with the anatomical finding depicted at the time of the other radiological evaluations, as described above. Furthermore, presence of voiding and storage symptoms at a younger age for long time duration urged us to look for the presence of functional voiding problem that could contribute to his infertility. Surgical management consisted of valve ablation using the hook of the resectoscope. Our patient reported improved urinary flow and ejaculation, followed by successful full-term pregnancy.

The abnormal urogenital anatomical findings were recognized after the initial radiological evaluation of obstructive azoospermia with the MRI, which showed a thick bladder wall, and the initial office cystoscopy, which revealed an abnormal prostatic urethra and severe bladder trabeculations. Subsequently, the findings of 
the VCUG obligated us to repeat the cystoscopy to look explicitly for any obstructive valves, which was confirmed.

The PUVs result in a wide spectrum of disease severity, ranging from incompatibility with life to a minimal impact that may not manifest during infancy. ${ }^{1}$ The management of PUVs remains a clinical challenge, requiring long-term management that differs among patients, based on severity and management time. Our patient presented with primary infertility with mild lower urinary tract symptoms, which were discovered due to attention to fertility. In fact, most of the reported cases of delayed presentation with PUVs are from countries where healthcare services are limited, with a lack of optimal programs for antenatal care. ${ }^{5}$

Taskinen et $\mathrm{al}^{6}$ reported adult sexual function among patients with PUVs. The patient and control groups in their cohort were similar in term of erectile function, except for patients with higher age due to the presence of secondary comorbidities to PUVs, such as renal impairment with chronic kidney disease, hypertension and renal transplants. However, their report was limited to a small sample size. Furthermore, the PUV group showed difficulties in achieving orgasms and reported ejaculatory problems. Nevertheless, paternity and ejaculatory problems were similar to those of the general population, except in the older age groups, which cannot be explained up to date. However, many factors could explain the sexual dysfunction among PUV patients, including renal impairments, embryological malformations of the prostate and seminal vesicles, chronic urinary reflux to ejaculatory ducts and recurrent epididymo-orchitis and prostatitis. ${ }^{7}$ Our patient was investigated for retrograde ejaculation as part of low ejaculate volume workup which revealed no sperms in the post-ejaculation urine analysis. However, based upon previous literature about the pathophysiology of the valve obstruction, ${ }^{7}$ chronic urine reflux to the genital organs secondary to obstruction might contribute to the abnormal environment for sperm storage and seminal fluid production.
The present case supports the wide spectrum of causes of male infertility that mandate detailed clinical evaluations. Systematic assessment and management of all urological complaints, including minor details, are essential to optimize patient condition for any hidden post-testicular urological factor of male infertility. In our report, the etiological factor in our patient was not diagnosed initially due to its rare occurrence at this age. However, this report addresses the diagnostic dilemma that could happen during infertility evaluation.

Obstructive azoospermia could result from obstruction at different levels in the urogenital tract. The high index of suspicion for PUVs should be considered for different age groups, especially for clinical presentation and radiological investigations suggestive of PUVs. In this patient, the associated urogenital tract abnormalities, including associated anatomical genital abnormalities and seminal fluid production, with PUV were surgically correctable and considered not to be barriers to achieve fertility after valve ablation.

\section{References}

1. Atwell JD. Posterior urethral valves in the British Isles: a multicenter B.A.P.S. review. J Pediatr Surg 1983; 18: 70-74.

2. Schober JM, Dulabon LM, Woodhouse CR. Outcome of valve ablation in late-presenting posterior urethral valves. BJU Int 2004; 94: 616-619.

3. Mahadik P, Vaddi SP, Godala CM, et al. Posterior urethral valve: Delayed presentation in adolescence. Int Neurourol J 2012; 16:149-152.

4. Font MD, Pastuszak AW, Case JR, et al. An infertile male with dilated seminal vesicles due to functional obstruction. Asian J Androl 2017; 19: 256-257.

5. Ikuerowo SO, Balogun BO, Akintomide TE, et al. Clinical and radiological characteristics of Nigerian boys with posterior urethral valves. Pediatr Surg Int 2008; 24: 825-829.

6. Taskinen S, Heikkila J, Santtila P, et al. Posterior urethral valves and adult sexual function. BJU Int 2012; 110: 392-396.

7. Schober JM, Dulabon LM, Gor RA, et al. Pyospermia in an adult cohort with persistent lower urinary tract symptoms and a history of ablated posterior urethral valve. J Pediatr Urol 2010; 6: 614-618. 